\title{
Models of classical Be stars with gravity darkening
}

\author{
Meghan A. McGill, T. A. Aaron. Sigut and Carol E. Jones \\ Department of Physics and Astronomy, The University of Western Ontario, \\ London, Ontario, Canada, N6A 3K7 \\ emails: mmcgill8@uwo.ca, asigut@uwo.ca, cejones@uwo.ca
}

\begin{abstract}
Classical Be stars are rapidly rotating, hot stars that possess an equatorial disk formed from gas released by the central star. The mechanism driving the stellar mass loss has yet to be fully explained, but the rapid rotation of the central B star is believed to be crucial. Rapid rotation also produces gravity darkening, and we have now extended our disk models to include these effects. In this contribution, we focus on the effect of gravity darkening on the thermal structure of a circumstellar disk.
\end{abstract}

Keywords. stars: emission-line, Be, circumstellar matter, stars: rotation

\section{Introduction}

A Be star disk re-processes the stellar radiation from the central star producing an emission line spectrum, particularly in the $\mathrm{H} \alpha$ line of hydrogen. A continuum excess is observed from the infrared to the radio, and Be systems can also exhibit linear polarization caused by electron scattering in the flattened disk (Porter \& Rivinius 2003). The major focus of Be star research is to explain how these disks are formed.

In a rapidly rotating star, the centrifugal acceleration causes the magnitude of the local gravity to decrease towards the equator. This distorts the shape of the star, making the radius larger at the equator than at the pole, and it also produces a decrease in the local temperature near the equator and an increase near the pole (Collins, 1966). This is illustrated in Fig. 1. The critical rotation velocity, $v_{\text {crit }}$, is defined as the equatorial rotation speed at which the equator is unbound, and at critical rotation, the equatorial radius is 1.5 times the polar radius. While the changes in the central star due to rapid rotation are well known, there has been little systematic work on how rapid rotation changes the temperature structure of the circumstellar disk.

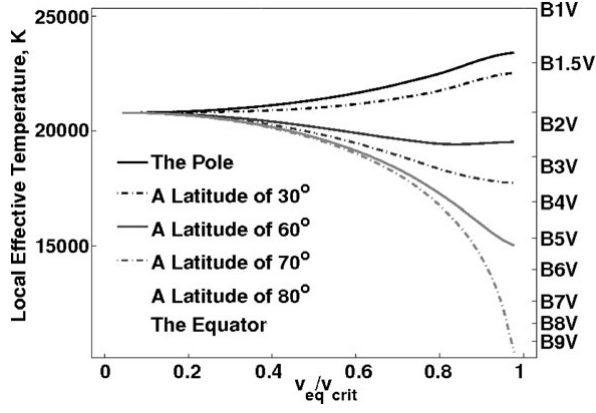

Figure 1. The divergence in the stellar surface temperatures at different latitudes with increasing rotation.

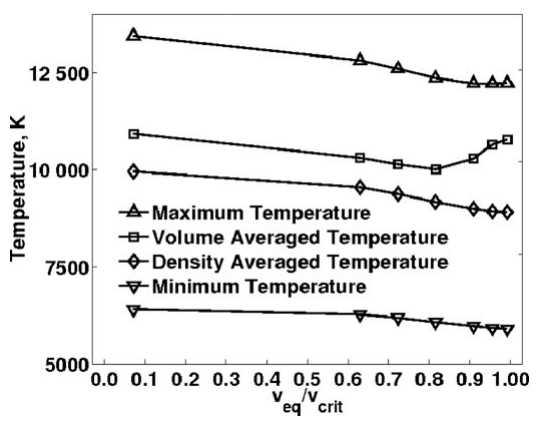

Figure 2. The change in several disk temperature diagnostics with increasing rotation. 

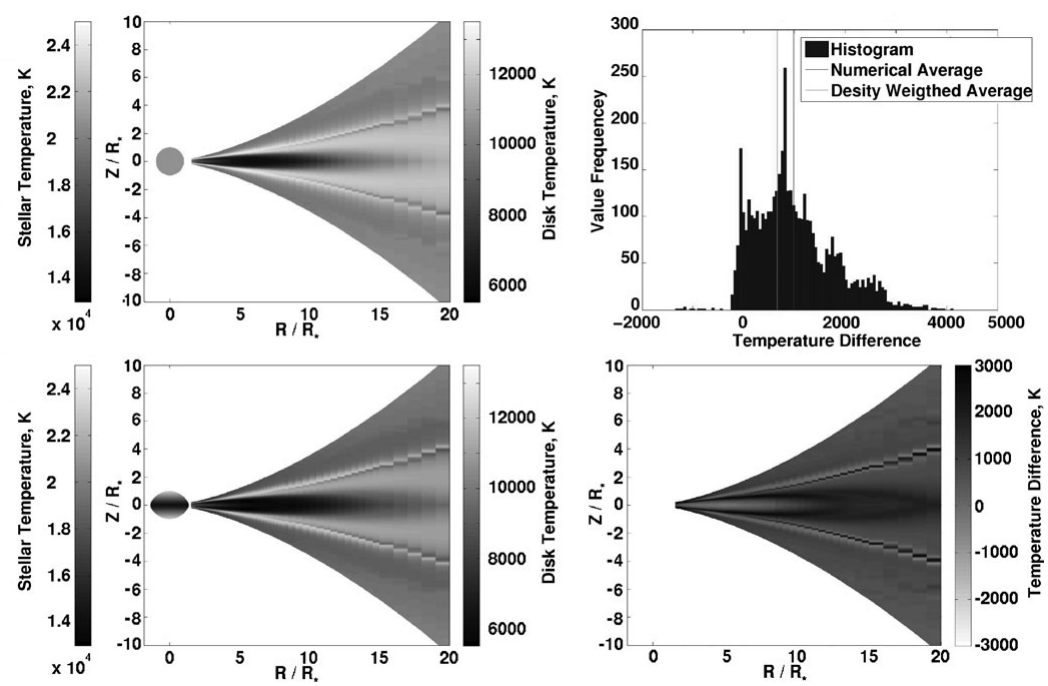

Figure 3. Top left: The temperature structure of a star and disk in the non-rotating case. Bottom left: The temperature structure of a star and disk for a star rotating at $v_{\text {frac }}=0.90$. Top right: A histogram of the temperature differences between the two models. Positive numbers indicate that the non-rotating model is hotter. Bottom right: The temperature differences in the disk between the non-rotating model and the rotating model.

\section{Calculations}

The BEDISK code of Sigut \& Jones (2007) has been modified to include gravity darkening. To illustrate these effects, we have calculated the thermal structure of a disk with an equatorial density of $\rho(R)=5 \cdot 10^{-11}\left(R_{*} / R\right)^{3.5} \mathrm{~g} \mathrm{~cm}^{-3}$ and of solar composition. The central star is assumed to be a B2V star with $R_{p}=5.33 R_{\odot}$ and $M=9.11 M_{\odot}$. The temperature structure of this disk was computed for rotation rates ranging from $0 \%$ to $99 \%$ of the critical rotation rate.

\section{Temperature Results}

The bulk of the disk becomes cooler as stellar rotation increases. This is shown in Fig. 2 and Fig. 3. The decrease in the average disk temperature is $\approx 800 \mathrm{~K}$ as $v_{\text {frac }}$ increases to 0.8. With faster rotation, the increasing temperature of the stellar pole begins to provide additional heating for the disk. At high rotation rates the density-averaged temperature continues to decrease, but the volume-averaged temperature increases as shown in Fig. 2. A detailed comparison of the disk temperature structure between a non-rotating star and one rotating at $v_{\text {frac }}=0.9$ is shown in Fig. 3. The inner cool core experiences no significant temperature change, but it does grow larger. The outer portions of the disk and regions far from the mid-plane decrease in temperature. The most interesting behaviour is in the hot sheet of material above the cool core, this sheet moves higher up in the disk, becomes narrower and increases slightly in temperature. More results, including those from additional spectral types, will be presented in McGill et al. 2010 (in preparation).

\section{References}

Collins, II, G. W. 1966, ApJ 146, 914

Porter, J. M. \& Rivinius, T. 2003, PASP, 115, 1153

Sigut, T. A. A. \& Jones, C. E. 2007, ApJ, 668, 481 